\title{
Frühling 2006 in Bangalore
}

\author{
Roland Schauer
}

\begin{abstract}
The buildings of the Indian Institute of Science in Bangalore (South India) are distributed in a large park in which a great variety of tropical plants, especially trees, are growing. Many animals, especially birds inhabit this forest-like area. At the end of February 2006 the park becomes impressively colourful, in spite of the natural dryness of the spring, due to the white, yellow, pink, red and blue flowers of many shrubs, but mainly of various Tabebuia, Jacaranda and Cassia tree species.
\end{abstract}

\section{Zusammenfassung}

Die Gebäude des Indian Institute of Science im südindischen Bangalore sind in einem großen Park verstreut, der eine Vielzahl von tropischen Pflanzen beherbergt. Der waldähnliche Park wird auch von zahlreichen Tieren, insbesondere Vögeln, belebt. Ende Februar 2006 zeigte sich das Gelände trotz großer Trockenheit in schönem Frühlingsflor, wobei vor allem Tabebuia-, Jacaranda- und Cassia-Bäume ein eindrucksvoll buntes Bild lieferten.

\section{Einleitung}

Bangalore liegt in Südindien am Rande der Tropen. In der Stadt, die sich auf der 800 m hohen Deccan-Hochebene ausdehnt, herrscht ein relativ trockenes, nicht zu heißes und sehr angenehmes Klima. Die Stadt hat über 4 Millionen Einwohner und gilt als die sauberste und wohlhabendste Stadt Indiens. Dazu trägt vor allem ihre Seiden- und Computer-Industrie bei ('Silk and Silicon Capital').
Auf dem Forschungs-Campus befindet sich ein großer, urwaldartiger Park. Er ist gleichzeitig ein Vogelschutzgebiet, in dem ca. 150 Vogelarten leben.

\section{Pflanzen im Park}

Die meisten Wege im Park sind von ausladenden, oft schirmartigen Bäumen beschattet. Einige Laubbäume waren Ende Februar allerdings noch kahl und andere warfen gerade ihre gelben oder braunen Blätter sowie ihre Früchte ab. Die

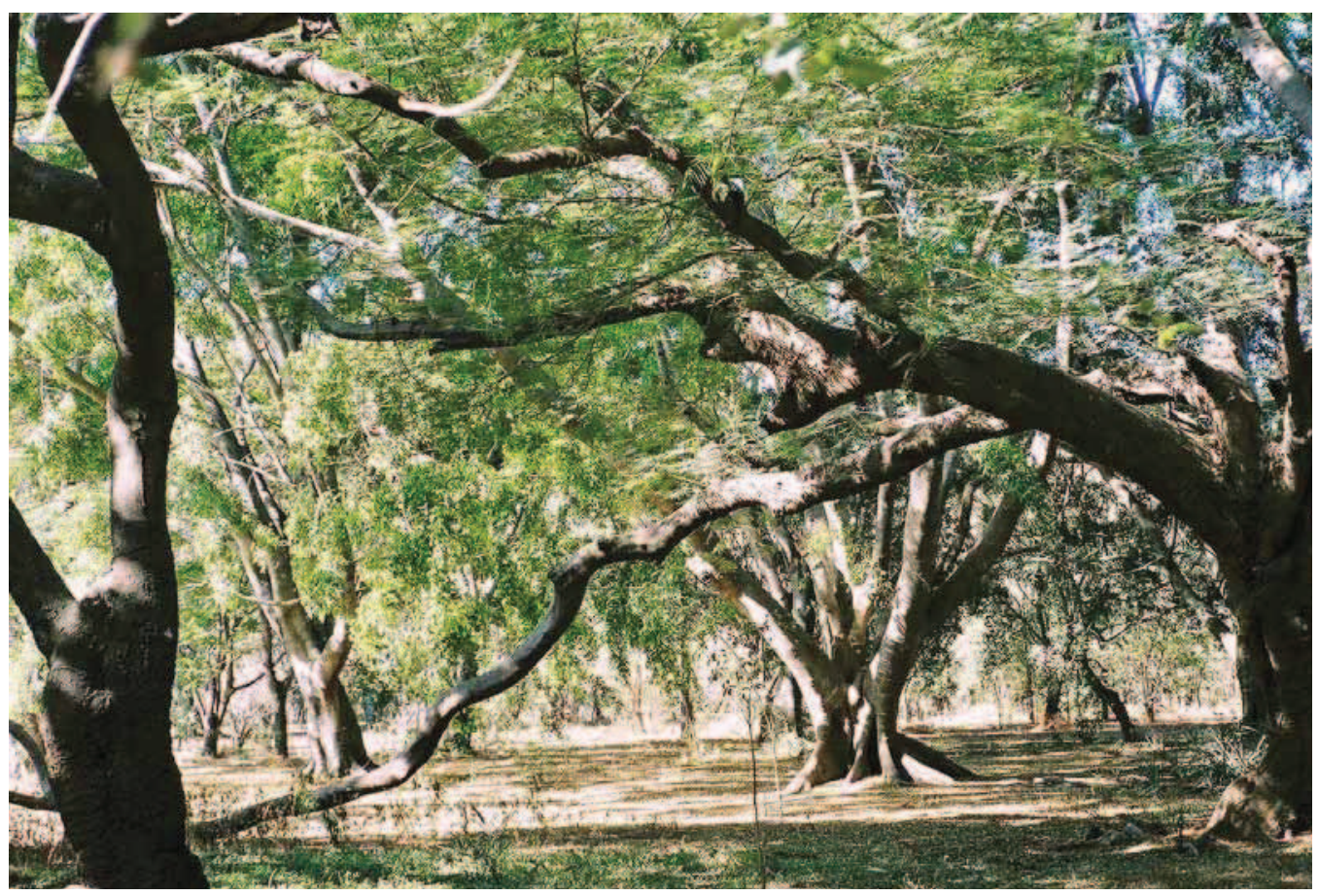


teilweise riesigen und recht alten Bäume und Sträucher des Parks stammen überwiegend aus Indien, manche aber auch aus anderen tropischen Ländern. In der Trockenzeit waren viele Pflanzen der Krautschicht verwelkt. An kahlen Stellen trat dann die für tropische Gebiete so typische leuchtend rotbraune Farbe des Bodens hervor.

Im Park konnte ich bis $60 \mathrm{~m}$ hohe schlanke Araukarien betrachten. $\mathrm{Zu}$ sehen waren aber auch sehr breit ausladende Bäume mit dicht belaubten Kronen (z. B. Schirmakazien, Regen- und Jacarandabäume). Besonders beeindruckend waren für mich die immergrünen, bis $40 \mathrm{~m}$ hohen Feigenbäume mit ihren ausladenden Ästen und Luftwurzeln, die auch als Stützen dienen. Die Blütenpracht der Bäume und Sträucher, die verschiedenen Düfte sowie das Vogelgezwitscher machten den Besuch des Parks zu einem unvergleichlichen Erlebnis. Üppige Blütenpracht gab es in Weiß, Gelb, Orange, Rosa, Rot, Blau oder Violett, wobei intensives Gelb die dominierende Farbe war. Sehr auffällig leuchteten die Blüten des Yellow Silk CottonBaumes (Cochlospermum religiosum), eines traditionellen Tempelbaumes. Beachtenswert waren auch die $30 \mathrm{~m}$ hohen Exemplare von Tabebuia argentea mit silbrigen Blättern und leuchtend gelben Blüten. Ihre flachen, flügelförmigen Früchte erinnern an Ahornfrüchte. In Indien gelten die Blüten dieses Baumes als Frühlingsboten. Über 4000 Exemplare von Tabebuia argentea wurden in Bangalore als Straßenbäume gepflanzt. Ihre abgefallenen, bis $7 \mathrm{~cm}$ breiten und langen, fünfzipfeligen, trichterförmigen Blüten bildeten einen gelben Teppich unter den Bäumen. Die riesigen Jacarandabäume (Jacaranda acutifolia) erzeugten einen blauen, intensiv duftenden Teppich aus abgefallenen Blüten. Zur Farbenpracht des Parks trugen auch die dunkelrosa gefärbten, trompetenförmigen, etwas kleineren Blüten von Tabebuia avellanedae

Abb. 1 (Seite 74): Birkenfeigen-Hain (Ficus benjamina).

Abb. 2 (oben): Tabebuja argentea.

Abb. 3 (Mitte): Jacaranda acutifolia.

Abb. 4 (unten): Tabebuia avellanedae.
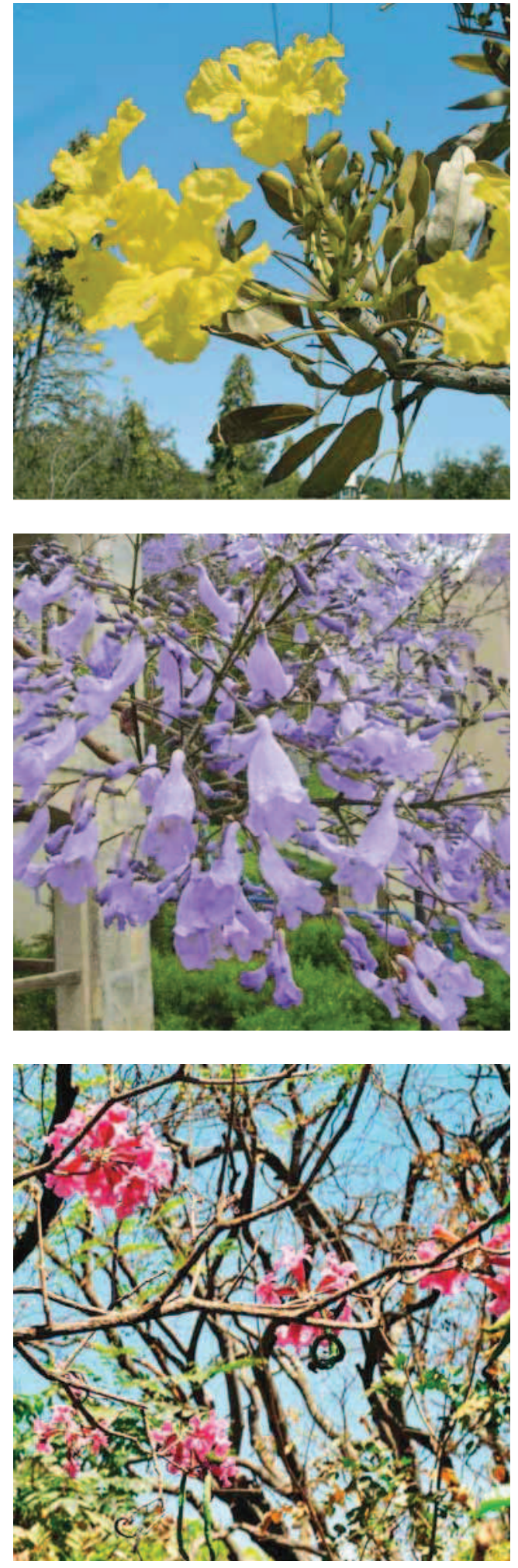

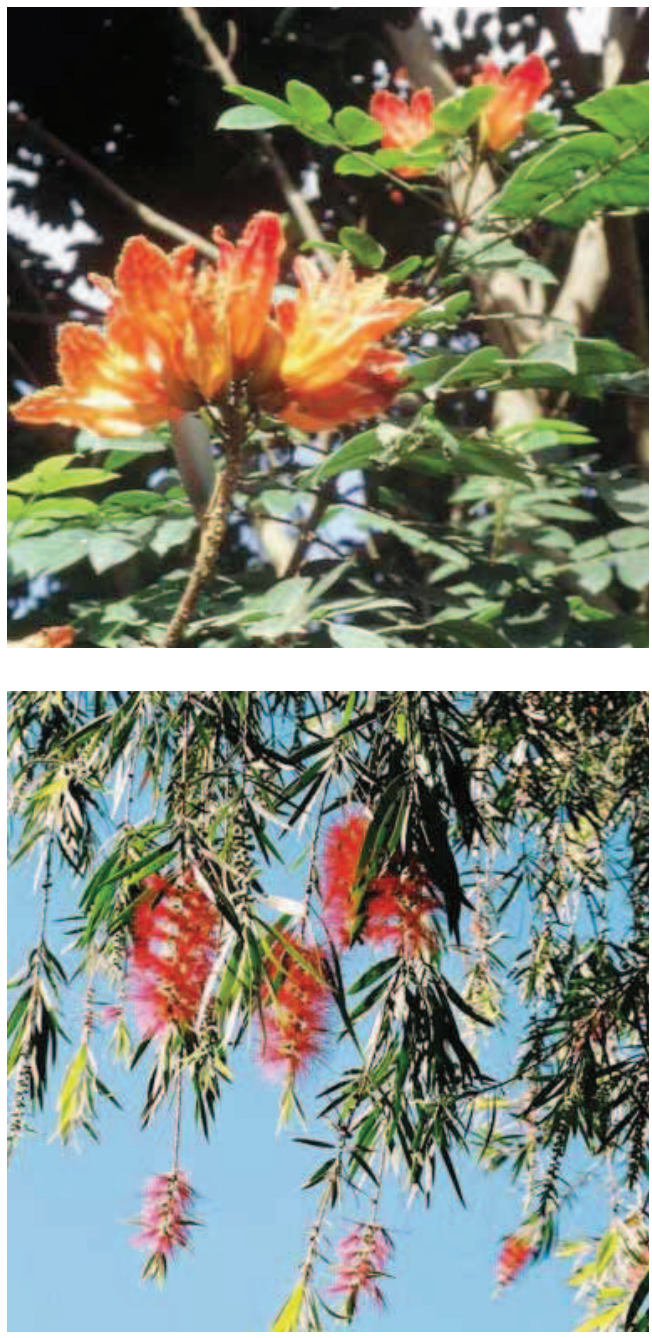

bei. Leider waren die Afrikanischen Tulpenbäume (Spathodea campanulata) mit ihren rot bis gelbrot leuchtenden Blüten fast verblüht. Es muss ein beeindruckendes Bild sein, wenn etwas früher im Jahr ihre Blütenpracht das Stadtbild bestimmt.

Beim Spaziergang durch den Park lohnt es sich auch, auf verschiedene Geräusche zu achten. Die langen, trockenen Hülsen der Cassia flattern und klappern im Wind. Elegant bewegen sich im Luftzug auch die hängenden Zweige des australischen Pinselstrauches (Callistemon lanceolatus) mit seinen feuerroten Blüten, deren Staubfäden die Schauwirkung übernehmen. Beachtenswert war auch der Leberwurstbaum
(Kigelia africana) aus trockenen Gebieten Afrikas. Seine Blüten hängen an langen Trieben aus der Krone heraus und sind damit für Blütenbesucher leicht zugänglich. Sie produzieren reichlich Nektar und werden von Fledermäusen, aber auch von Vögeln und Insekten besucht. Im Februar hingen über $50 \mathrm{~cm}$ lange und $4 \mathrm{~kg}$ schwere Früchte an den Bäumen. Sie sind für den Menschen ungenießbar.

Zusätzlich schmücken den Park zahlreiche Bougainvillea-Pflanzen, deren Blüten die Farben von Rot bis Gelb zeigen. Sie klettern oft hoch in Baumkronen hinein oder sind in Form geschnitten. Vor dem Kongressgebäude blühte gerade der Ashok-Baum (Saraca indica). Der Volksname bezieht sich auf den König Аsнок, der vermutlich von ca. 268 v. Chr. -233 v. Chr regierte und die ersten buddhistischen Schriften in Höhlengestein meißeln ließ.

Der Regenbaum (Pithecolobium saman) ist ein weit ausladender Baum aus der Familie der Leguminosen mit büschelig angeordneten, rosa oder weißgelben Blüten. Bei Regen und Dunkelheit falten sich die Fiedern zur Mittelrippe hin zusammen. Besonders dekorativ ist auch Caesalpinia pulcherrima, die ihre zarten rotgelben Blüten in den blauen Himmel streckt. Weitere erwähnenswerte Gehölze im Park sind beispielweise Erythrina indica, Bauhinia spec., Cassia fistula, C. nodosa, Milletia peguensis, Ficus krishnae und $F$. benghalensis.

Diese Vielfalt haben wir dem Landschaftsgärtner Gustav Hermann Krumbiegel (18651956) aus Dresden zu verdanken, der in diesem Park wirkte.

\section{Tiere im Park}

Der Park ist auch Lebensraum für zahlreiche Wildtiere. Wer die Vögel des Parks sehen möchte, wird vielleicht etwas enttäuscht sein, denn die meisten sind mehr zu hören als zu sehen. Nach meist intensivem Morgenrot, gefolgt

Abb. 5 (oben): Afrikanischer Tulpenbaum (Spathodea campanulata).

Abb. 6 (unten): Flaschenbürstenbaum (Callistemon lanceolatus). 
von einer prächtigen Sonne gegen 7 Uhr, beginnen viele Singvögel Strophen vorzutragen. Kurz nach Sonnenuntergang, wenn der Park für kurze Zeit in ein unwirkliches rotes bis violettes Dämmerlicht getaucht ist, klingt der Gesang der Vögel besonders intensiv.

Am Abend laufen Geckos an den Wänden im Flur des Gästehauses und jagen Insekten. Es gibt viele kleine Nachtfalter, zum Glück aber keine Stechmücken. Außerdem begeistern herrliche Schmetterlinge, die oft sehr farbintensiv sind wie der blau leuchtende Odysseus-Falter (Papilio ulysses). Daneben lassen sich große bräunliche Libellen und grünliche Heupferde beobachten. Viel Freude bereiteten auch die Affen, vermutlich die in Indien weit verbreiteten Bonnet-Makaken (Macaca radiata). Auch Streifenhörnchen und viele verwilderte Hunde gehören zur Fauna dieses wunderschönen tropischen Parks.

Abb. 7 (oben): Ashok-Baum (Saraca indica).

Abb. 8 (unten): 'Peacock Flower Bush' (Caesalpina pulcherrima).
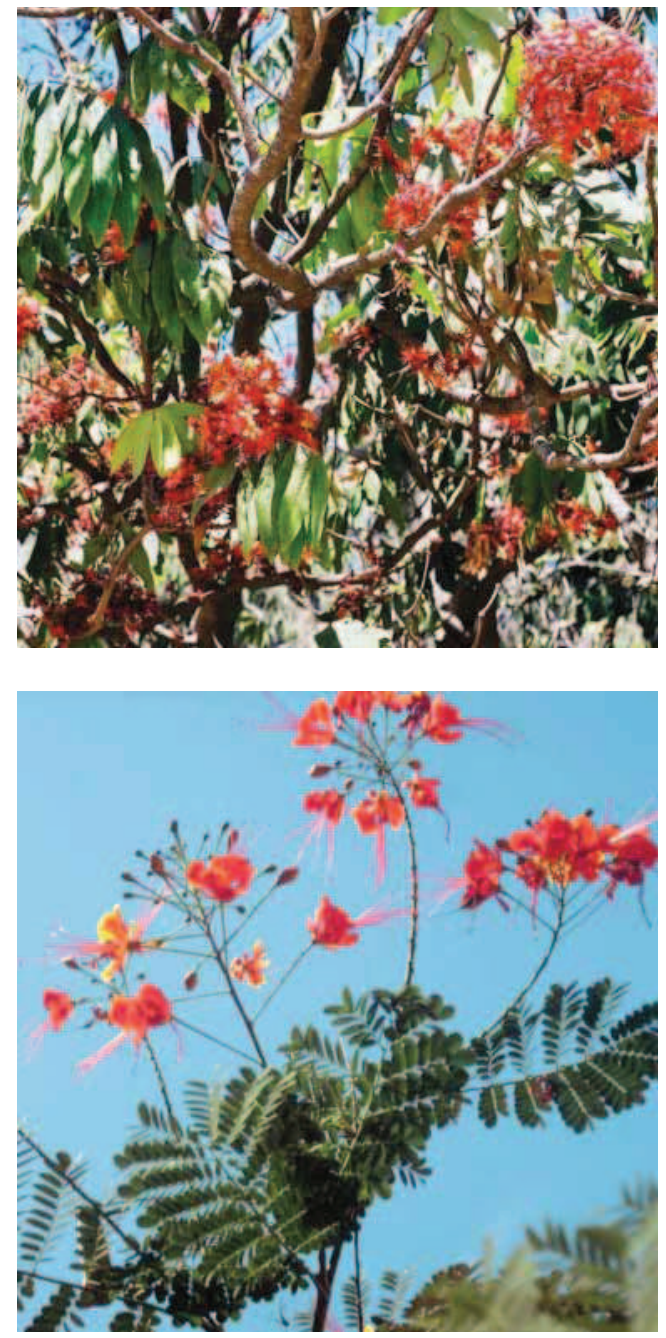